\title{
Effect of an Inclined Magnetic Field on Steady Poiseuilleflow between Two Parallel Porous Plates
}

\author{
D.R.Kuiry ${ }^{1}$ and Surya Bahadur ${ }^{2}$ \\ ${ }^{I}$ P.G. Department of Mathematics, Kolhan University, Chaibasa-833202, INDIA \\ ${ }^{2}$ Department of Mathematics, R.V.S.College of Engineering and Technology, Jamshedpur-831012,
} INDIA

\begin{abstract}
The present work is to investigate the magnetohydrodynamic behaviour of two dimensional Poiseuille flow under the influence of an inclined magnetic field and constant pressure gradient for a viscous, incompressible and electrically conducting fluid between two infinite parallel plates of which the lower plate is taken to be porous. The solution of the fluid flow governing equation has been obtained by analytical method and expressed in terms of Hartmann number. The fluid flow profiles are represented through graphs for different values of Hartmann number with different angles of inclination for injection/suction. The effects of the other parameters such as pressure gradient, inclined magnetic field and suction/injection on the flow are also studied graphically. The resulting equation of motion has preferably been solved by the method of variation of parameters.
\end{abstract}

Key words: Poiseuille flow; Porous plate; Conducting fluid; Pressure gradient; Inclined magnetic field; Hartmann number.

\section{Introduction}

Magnetohydrodynamics studies the motion of an electrically conducting fluid in the presence of magnetic field. Liquid metals such as mercury, molten iron, and ionized gases (plasma) are treated as electrically conducting fluids. The subject magnetohydrodynamics has been studied by Alfve'n [1]. It is the well-known results of electrodynamics that when an electrically conducting fluid moves in a magnetic field, electric currents are induced and these currents lead to cause the magnetohydrodynamic (MHD) generators to produce electricity with an arrangement of some other devices.

The physical interest that one can think of through a problem of fluid flow due to an inclined magnetic field is found through the application of it, mainly in the study of the design of MHD power generator and other applications are observed in MHD pumps, accelerators and so on. So the analysis of such problem has been studied by a number of researchers e.g. Water house and Kingston [13], Shercliff [10], Kazuyuki [6], Kurri[7], Ganesh and Krishnambal[5], Singh and Ram [12], Kuiry [8], Bear [2], Mahato and Kuiry [9] and many others under different conditions and situations.

In the present work the focus is given on the steady magnetohydrodynamic two-dimensional Poiseuille flow between two infinite parallel plates of which the lower plate is assumed to be porous subject to a constant, inclined uniform magnetic field to the fluid flow under a constant pressure gradient to determine the effect on the fluid flow .The governing differential equation is solved by analytical method and the solution expressed in terms of Hartmann number. The graphical representation for velocity curves for the cases of injection/ suction with different Hartmann numbers at different values of the inclined angles is exhibited.

\section{MathematicalFormulation Of The Problem}

Let an electrically conducting fluid move with the velocity $\vec{V}$ and at right angles to this flow, the magnetic field of strength $\vec{B}$ is applied. Let the fluid attain the steady state condition i.e the flow variables are independent of time t. On account of the interaction of the velocity and magnetic field, an electric field $\vec{E}$ is induced at right angles to both $\vec{V}$ and $\vec{B}$ given by the following manner:

$$
\vec{E}=\vec{V} \times \vec{B}
$$

The current density Jinduced in the conducting fluid is given by Ohm's law [11] in the form:

$$
\vec{J}=\sigma(\overrightarrow{\mathrm{V}} \times \overrightarrow{\mathrm{B}})
$$

$\sigma$ being the electrical conductivity and with an induced current a force known as Lorentz is provided in the form:

$$
\overrightarrow{\mathrm{F}}=\vec{J} \times \overrightarrow{\mathrm{B}}
$$


This occurs because, as an electric generator, the conducting fluid cuts the magnetic lines of force. This force $\overrightarrow{\mathrm{F}}$ is parallel to the field velocity $\overrightarrow{\mathrm{V}}$ but in opposite direction. The applications, such as the MHD pump, MHD generator, and electromagnetic flow meter, are observed through the flow under uniform magnetic field.

Now let an electrically conducting, viscous, incompressible fluid move between two infinite parallel plates separated by a distance $2 \mathrm{~d}$ and both the plates are at rest .Such a flow indicates a plane Poiseuille flow with a constant pressure gradient.

Let the origin be at the centre of the channel with $x$-axis parallel and y-axis perpendicular to the channel walls respectively. Since the plates are of infinite length, all the variables except the fluid pressure are functions of $\mathrm{y}$ only. The lower plate is at $\mathrm{y}=-1$ and the upper plate is at $\mathrm{y}=1$. Further the fluid flow is practically horizontal along the $\mathrm{x}$-axis .If $\mathrm{u}$ and $\mathrm{v}$ are the components of the velocity $\overrightarrow{\mathrm{V}}$, for an incompressible fluid the equation of continuity $\nabla$. $(u, v)=0$ takes the form $\frac{\partial v}{\partial y}=0$ and this yields $v=$ constant $=v_{0}$ which indicates that $\mathrm{u}=\mathrm{u}(\mathrm{y})$

Now, if we take $\mathrm{v}_{0}$ to be the characteristic velocity perpendicular to the fluid flow to maintain a steady flow at a constant pressure gradient, this $\mathrm{v}_{0}$ at the lower plate is the one which will maintain a steady fluid flow against suction and injection.

The governing differential equations are expressed in the simplified form as below:

and

$$
\mathrm{v}_{0} \frac{\partial \mathrm{u}}{\partial \mathrm{y}}=\frac{\mathrm{F}_{\mathrm{x}}}{\rho}-\frac{1}{\rho} \frac{\partial \mathrm{p}}{\partial \mathrm{x}}+v \frac{\partial^{2} \mathrm{u}}{\partial \mathrm{y}^{2}}(4)
$$

$$
0=-\frac{1}{\rho} \frac{\partial \mathrm{p}}{\partial \mathrm{y}}
$$

where $F_{x}$ is the $x$-component of the magnetic field and $v$ is the kinematic viscosity defined as $v=\frac{\mu}{\rho}, \mu$ is the effective viscosity of the porous region and $\rho$ is the fluid density.

The equation (5) implies that the fluid pressure $p=p(x)$ and the equation (4) takes the form:

or,

$$
\mathrm{v}_{0} \frac{\partial \mathrm{u}}{\partial \mathrm{y}}=\frac{\sigma}{\rho} \mathrm{B}_{0}{ }^{2} \mathrm{u}-\frac{1}{\rho} \frac{\partial \mathrm{p}}{\partial \mathrm{x}}+v \frac{\partial^{2} \mathrm{u}}{\partial \mathrm{y}^{2}}
$$

or,

$$
\mathrm{v}_{0} \frac{\mathrm{du}}{\mathrm{dy}}=-\frac{\sigma}{\rho} \mathrm{B}_{0}{ }^{2} \mathrm{u}-\frac{1}{\rho} \frac{\partial \mathrm{p}}{\partial \mathrm{x}}+v \frac{\mathrm{d}^{2} \mathrm{u}}{\mathrm{dy}}
$$

where $\theta$ is the angle made by $\vec{V}=$ uî and $\vec{B}=B_{0} \hat{\jmath}$; $\hat{\text { inand }}$ jbeing the unit perpendicular vectors along $\mathrm{x}$ and $\mathrm{y}$ axes respectively and consequently the equation (6) shows that the fluid is under the influence of an inclined magnetic field.

Differentiating (6) with respect to $x$, it finds that $\frac{\mathrm{d}^{2} \mathrm{p}}{\mathrm{dx}^{2}}=0$ and so $\frac{\mathrm{dp}}{\mathrm{dx}}=$ constant $=\mathrm{P}$ (suppose)

The equation (6) is modified as:

$$
\frac{\mathrm{d}^{2} \mathrm{u}}{\mathrm{dy} \mathrm{y}^{2}}-\frac{\mathrm{v}_{0}}{v} \frac{\mathrm{du}}{\mathrm{dy}}-\frac{\sigma}{\mu} \mathrm{B}_{0}{ }^{2}\left(\operatorname{Sin}^{2} \theta\right) \mathrm{u}-\frac{1}{\mu} \mathrm{P}=0(7)
$$

which is, general in the sense that the two fields can be assessed at an angle $\theta$ for $0 \leq \theta \leq \pi$ with the boundary conditions $\mathrm{u}=0$ fory $= \pm \mathrm{d}$.

Let us put the following non-dimensional variables: $\quad \overline{\mathrm{x}}=\frac{\mathrm{x}}{\mathrm{d}}, \overline{\mathrm{y}}=\frac{\mathrm{y}}{\mathrm{d}}, \overline{\mathrm{p}}=\frac{\rho \mathrm{d}^{2}}{v^{2}}, \overline{\mathrm{u}}=\frac{\mathrm{ud}}{v}$

$\mathrm{g}=-\frac{\mathrm{P}}{\mu}, \lambda=\frac{\mathrm{v}_{0}}{v} \mathrm{~d}$, where $\lambda<0$ is for suction and $\lambda>0$ is for injection, $\mathrm{M}=\mathrm{M}^{*} \sin \theta$ or $\mathrm{M}=\mathrm{Ha} \cdot \sin \theta$, where $\mathrm{M}^{*}=\mathrm{Ha}=\mathrm{B}_{0} \mathrm{~d} \sqrt{\frac{\sigma}{\mu}}$ into the equation (7) and then dropping bars for convenience, it reduces to the form:

with the boundary conditions

$$
\frac{d^{2} u}{d y^{2}}-\lambda \frac{d u}{d y}-M^{2} u+g=0(8)
$$

$\mathrm{u}=0$ at $\mathrm{y}=1$ and $\mathrm{u}=0$ at $\mathrm{y}=-1$

\section{Solution Of The Problem}

The governing fluid flow equation (8) is an ordinary differential equation with constant co-efficient along with the boundary conditions (9) and so it can be solved by the method of variation of parameter $[3,11]$ to yield the solution of the type:

$$
\mathrm{u}(\mathrm{y})=-\frac{\mathrm{g}}{\mathrm{m}_{1} \mathrm{~m}_{2}}+\mathrm{c}_{1} \mathrm{e}^{\mathrm{m}_{1} \mathrm{y}}+\mathrm{c}_{2} \mathrm{e}^{\mathrm{m}_{2} \mathrm{y}}
$$

Where $m_{1}$ and $m_{2}$ are the roots of the auxiliary equation of obtained from the equation (8). Using the conditions (9), the values of the constants are obtained in the forms: 
$\operatorname{and}(11)$

$$
c_{1}=\frac{c}{m_{1} m_{2}}\left[\frac{\sinh \left(m_{2}\right)}{\sinh \left(m_{2}-m_{1}\right)}\right]
$$

$$
c_{2}=\frac{-c}{m_{1} m_{2}}\left[\frac{\sinh \left(m_{1}\right)}{\sinh \left(m_{2}-m_{1}\right)}\right]
$$

These values of the constants are used to find the solution of the governing equation (8) in terms of Hartmann number and expressed as:

$$
\mathrm{u}(\mathrm{y})=\frac{\mathrm{g}}{\mathrm{M}^{2}}\left[1+\frac{1}{\sinh (2 \varnothing)}\left\{\mathrm{e}^{\frac{\lambda}{2}(\mathrm{y}+1)}\{\sinh \{\phi(\mathrm{y}-1)\}\}-\mathrm{e}^{\frac{\lambda}{2}(\mathrm{y}-1)}\{\sinh \{\phi(\mathrm{y}+1)\}\}\right\}\right]
$$

where $\lambda<0$ : for suction and $\lambda>0$ :for injection:

$\varnothing=\sqrt{\left(\frac{\lambda}{2}\right)^{2}+M^{2}} \quad$, where $M=$ Ha. $\sin \theta$; Ha is the Hartmann number.

It determines the velocity field for different values of the Hartmann numbers.

\section{Discussion and Conclusion}

Taking $\mathrm{Ha}=1,1.5,2$ for the angle of inclination of $\theta=15^{\circ}$, keepingg $=1.2$ fixed, for the case of injection $\lambda=2$ (Fig.1) \& for the angle of inclination $\theta=30^{\circ}$ with the same parameters $g=1.2$ and $\lambda=2$ (Fig.2), the graphs have been drawn to study the velocity profile.

For the angle of inclination $30^{\circ}$ with suction parameter $\lambda=-2$ (Fig. 3) fordifferent values of $\mathrm{Ha}=1,1.5,2$; the graphs have been drawn to study the velocity profile.

Figures- $1 \& 2$ conclude that for high Hartmann numbers, the applied magnetic field intensity is higher resulting in decrease in the fluid velocity whenever there is an injection with a constant pressure gradient.

Figure -3 indicates that with an increase of Hartmann number, the applied magnetic field intensity is higher resulting in decrease in the fluid velocity even in the case of suction with a constant pressure gradient.

Figure- 4 indicates that the effect of increasing inclinations of the applied magnetic field reduces the velocity of the flow. The transversely applied magnetic field results in decreasing the fluid flow substantially. If the magnetic field is applied at a decreasing rate of inclination, the velocity of the fluid flow will be higher.

Figure-5 indicates that the fluid velocity goes on decreasing with an increase of injection parameter $\lambda$ and obviously the same effect will be observed in the case of suction too ( for negative values of $\lambda$ ).

Figure -6 shows that the fluid velocity is influenced by pressure gradient. An increase in the pressure gradient increases the velocity of the flow when the other parameters of flow are kept constant.

Table-1

\begin{tabular}{|c|c|c|c|}
\hline \multicolumn{4}{|c|}{ Table: $1 \theta=15^{\circ}, \lambda=2, \mathrm{~g}=1.2$ : Case of injection } \\
\hline $\mathrm{y}$ & $\mathrm{Ha}=1$ & $\mathrm{Ha}=1.5$ & $\mathrm{Ha}=2$ \\
\hline-1 & 0 & 0 & 0.128391 \\
\hline-0.75 & 0.133581 & 0.131360 & 0.245415 \\
\hline-0.5 & 0.257251 & 0.252175 & 0.346250 \\
\hline-0.25 & 0.365205 & 0.357065 & 0.422593 \\
\hline 0 & 0.447841 & 0.436985 & 0.460211 \\
\hline 0.25 & 0.489166 & 0.476708 & 0.434804 \\
\hline 0.5 & 0.462625 & 0.450650 & 0.304933 \\
\hline 0.75 & 0.324036 & 0.315814 & 0 \\
\hline 1 & 0 & 0 & \\
\hline
\end{tabular}




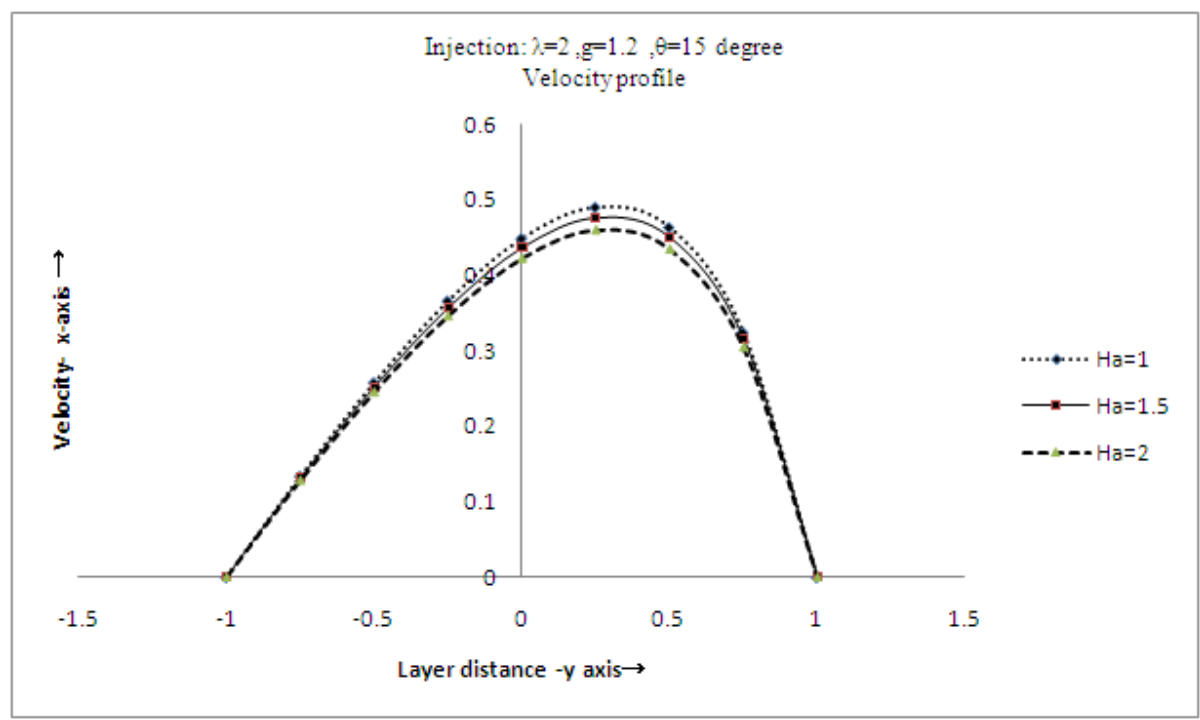

Figure1: $\theta=15^{\circ}, \lambda=2, \mathrm{~g}=1.2$ case of injection

Table-2

\begin{tabular}{|c|c|c|c|}
\hline \multicolumn{4}{|c|}{ Table: $2 \theta=30^{\circ}, \lambda=2, \mathrm{~g}=1.2$ : Case of injection } \\
\hline $\mathrm{y}$ & $\mathrm{Ha}=1$ & $\mathrm{Ha}=1.5$ & $\mathrm{Ha}=2$ \\
\hline-1 & 0 & 0 & 0.113017 \\
\hline-0.75 & 0.128829 & 0.230390 & 0.210748 \\
\hline-0.5 & 0.246419 & 0.321765 & 0.291245 \\
\hline-0.25 & 0.347858 & 0.390127 & 0.349888 \\
\hline 0 & 0.424733 & 0.423091 & 0.377281 \\
\hline 0.25 & 0.462663 & 0.399202 & 0.355383 \\
\hline 0.5 & 0.437158 & 0.280486 & 0.250433 \\
\hline 0.75 & 0.306547 & 0 & 0 \\
\hline 1 & 0 & & \\
\hline
\end{tabular}

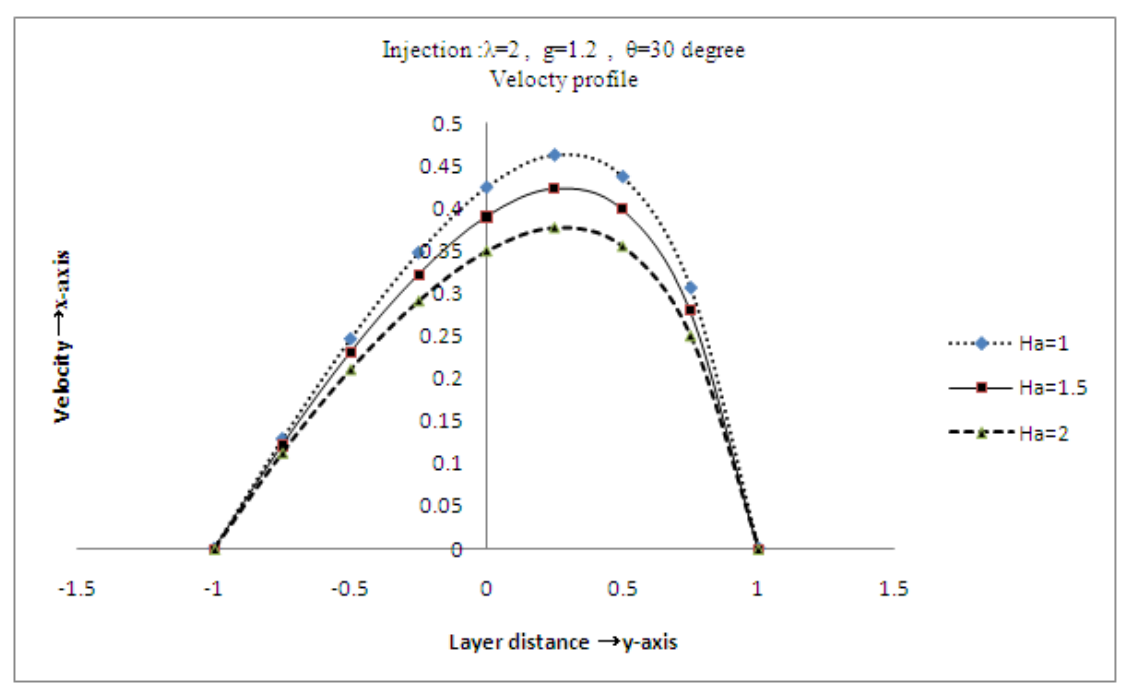

Figure2: $\theta=30^{\circ}, \lambda=2 \mathrm{~g}=1.2$ Case of injection

Table-3

\begin{tabular}{|c|c|c|c|}
\hline \multicolumn{4}{|c|}{ Table-3: $\theta=30^{\circ}, \lambda=-2, \mathrm{~g}=1.2,:$ Case of Suction } \\
\hline $\mathrm{y}$ & $\mathrm{Ha}=1$ & $\mathrm{Ha}=1.5$ & $\mathrm{Ha}=2$ \\
\hline-1 & 0 & 0 & 0 \\
\hline-0.75 & 0.306547 & 0.280486 & 0.250433 \\
\hline-0.5 & 0.437158 & 0.399202 & 0.355383 \\
\hline-0.25 & 0.462663 & 0.423091 & 0.377281 \\
\hline 0 & 0.424733 & 0.390127 & 0.349888 \\
\hline 0.25 & 0.347858 & 0.321765 & 0.291245 \\
\hline 0.5 & 0.246419 & 0.230390 & 0.210748 \\
\hline 0.75 & 0.128829 & 0.121595 & 0.113017 \\
\hline 1 & 0 & 0 & 0 \\
\hline
\end{tabular}




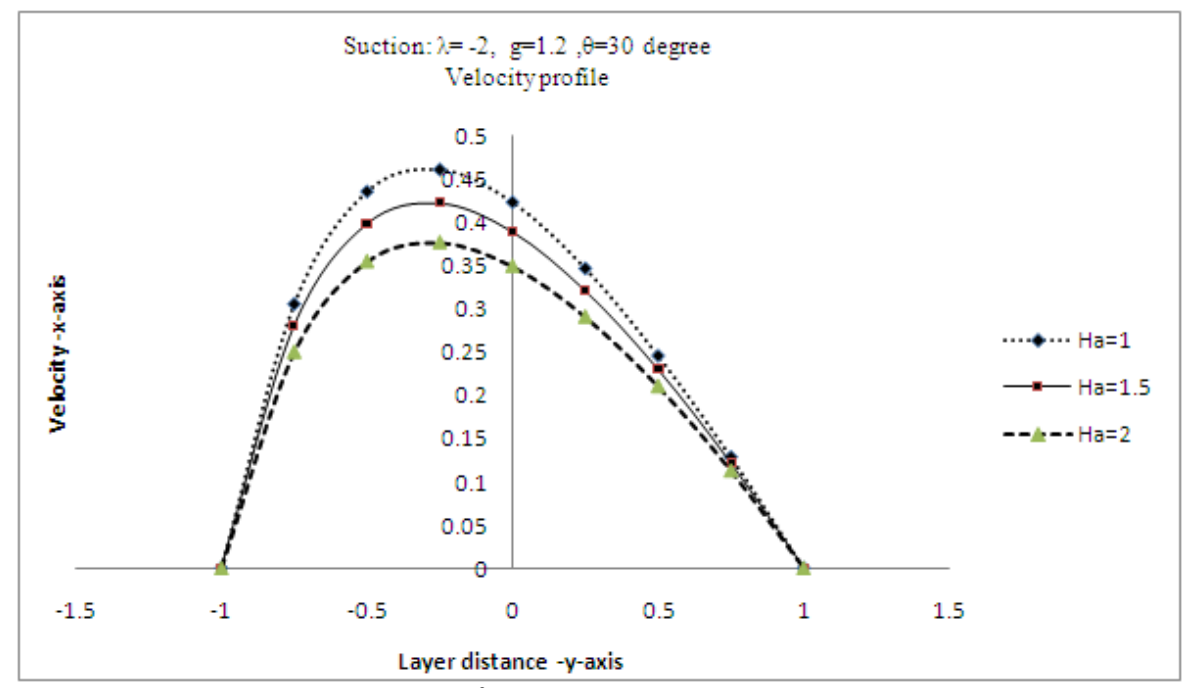

Figure-3: $\theta=30^{\circ}, \lambda=-2, \mathbf{g}=1.2$ Case of suction

Table-4

\begin{tabular}{|c|c|c|c|}
\hline \multicolumn{4}{|c|}{ Table-4: Velocity against magnetic inclinations $\theta$ : for $\lambda=2, \mathrm{Ha}=1.5, \mathrm{~g}=1.5, \mathrm{M}=\mathrm{Ha} \cdot \sin \theta$} \\
\hline $\mathrm{y}$ & $\theta=15$ & $\theta=30$ & $\theta=90$ \\
\hline-1 & 0 & 0 & 0 \\
\hline-0.75 & 0.164201 & 0.151989 & 0.118878 \\
\hline-0.5 & 0.315218 & 0.287547 & 0.213756 \\
\hline-0.25 & 0.44633 & 0.402207 & 0.286581 \\
\hline 0 & 0.546228 & 0.487659 & 0.336489 \\
\hline 0.25 & 0.595881 & 0.528864 & 0.357824 \\
\hline 0.5 & 0.56331 & 0.499004 & 0.335988 \\
\hline 0.75 & 0.394766 & 0.350609 & 0.238947 \\
\hline 1 & 0 & 0 & 0 \\
\hline
\end{tabular}

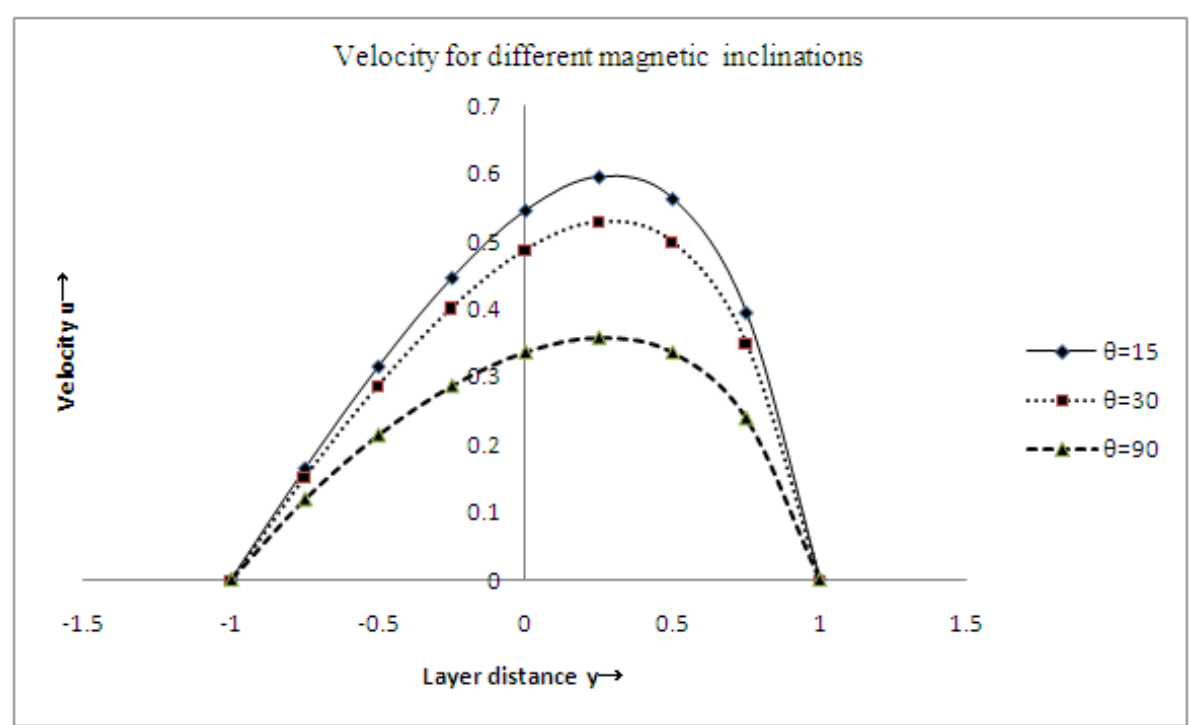

Figure4-Case of injection for velocity for the parameters $\lambda=2, \mathrm{Ha}=1.5, \mathrm{~g}=1.5, \mathrm{M}=\mathrm{Ha} . \sin \theta$ 
Table-5

\begin{tabular}{|c|c|c|c|}
\hline \multicolumn{4}{|c|}{ Table-5 : Velocity against different values of $\lambda: \quad \mathrm{g}=1.5, \theta=30^{\circ}, \mathrm{Ha}=1.5, \mathrm{M}=0.75$} \\
\hline $\mathrm{y}$ & $\lambda=2$ & $\lambda=2.5$ & $\lambda=3$ \\
\hline-1 & 0 & 0 & 0 \\
\hline-0.75 & 0.151989 & 0.13085 & 0.113717 \\
\hline-0.5 & 0.287547 & 0.251081 & 0.220403 \\
\hline-0.25 & 0.402207 & 0.357384 & 0.317801 \\
\hline 0 & 0.487659 & 0.442872 & 0.400651 \\
\hline 0.25 & 0.528864 & 0.493661 & 0.457053 \\
\hline 0.5 & 0.499004 & 0.482112 & 0.460423 \\
\hline 0.75 & 0.350609 & 0.35353 & 0.351709 \\
\hline 1 & 0 & 0 & 0 \\
\hline
\end{tabular}

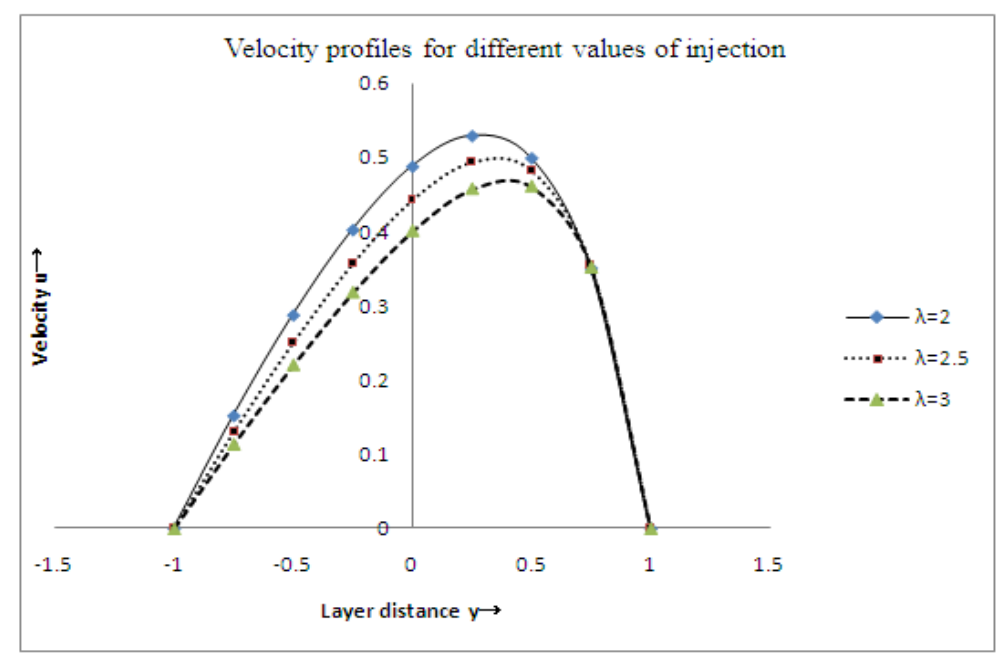

Figure5:Case of injection for velocity for the parameters $\mathrm{Ha}=1.5, \mathrm{~g}=1.5, \mathrm{M}=\mathrm{Ha} \cdot \sin \theta=0.75, \boldsymbol{\theta}=\mathbf{3 0}^{\circ}$

Table-6

\begin{tabular}{|c|c|c|c|}
\hline \multicolumn{4}{|c|}{ Table-6: Velocity against pressure gradient $\mathrm{g}: \lambda=2, \theta=30^{\circ}, \mathrm{Ha}=1.5, \mathrm{M}=0.75$} \\
\hline $\mathrm{y}$ & $\mathrm{g}=1.5$ & $\mathrm{~g}=2.5$ & $\mathrm{~g}=3.5$ \\
\hline-1 & 0 & 0 & 0 \\
\hline-0.75 & 0.151989 & 0.253315 & 0.354641 \\
\hline-0.5 & 0.287547 & 0.479245 & 0.938943 \\
\hline-0.25 & 0.402207 & 0.670345 & 1.137871 \\
\hline 0 & 0.487659 & 0.812765 & 1.234016 \\
\hline 0.25 & 0.528864 & 0.88144 & 1.164342 \\
\hline 0.5 & 0.499004 & 0.831673 & 0.818087 \\
\hline 0.75 & 0.350609 & 0.584348 & 0 \\
\hline 1 & 0 & 0 & \\
\hline
\end{tabular}

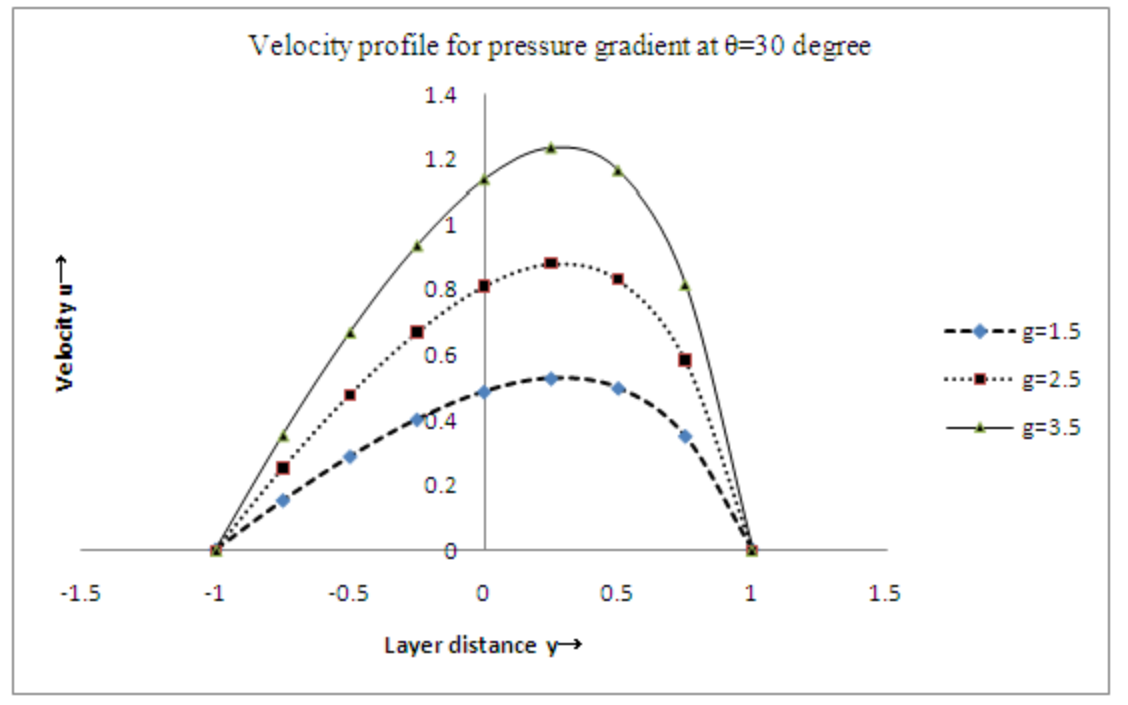

Figure6:Velocity against pressure gradient for the parameters: $\lambda=2, \theta=30^{\circ}, \mathrm{Ha}=1.5, \mathrm{M}=0.75$ 


\section{References}

[1]. H.Alfve'n. Existence of electromagnetic hydrodynamic waves, Nature, 150, No.3805 (1942), 405-406.

[2]. J.Bear, Dynamics of fluids in porous media, Dover (1988).

[3]. Richard Bronson \& Gabriel B Costa, Differential Equations, Thirdedition, Tata McGraw-Hill Education Private Limited, New Delhi

[4]. P.A.Davidson, An Introduction to Magnetohydrodynamics, First edition, Cambridge University Press, UK (2001).

[5]. S.Ganesh and S. Krishnambal, Unsteady MHD Stokes flow of viscous fluid between two parallel porous plates, J.Appl.Sci. 7(2007), 374-379.

[6]. U.Kazuyuki, Inertia effects on two- dimensional magnetohydrodynamic channel flow under traveling sine wave magnetic field, Phys.Fluids A, 3 No.12 (1991), 3107-3116.

[7]. D.R.Kuiry, Generalized porous wall "Couette- type" MHD flow, J .Indian Math .Soc .67, 1-2(2000), $117-121$.

[8]. D.R.Kuiry, On Hartmann -and -neutral point type MHD flow in a duct, J .Indian Math. Soc .78, No. 1-4 (2011), 79-85.

[9]. T.K.Mahato and D.R.Kuiry, Hydromagnetic flow of a viscous incompressible fluid over a naturally permeable bed, Indian J.Theo.Phys, Vol.47.No.2 (1999), 139-148.

[10]. A.Shercliff, Entry of conducting and non-conducting fluids in pipes, J.Math. Proc.of the Cambridge Philos. Soc.52 (1950)573-583

[11]. G.F.Simmons, Differential equations with Applications and Historical Notes., TMH,Ed. (1974), McGraw-Hill, Inc. New York.

[12]. C.B.Singh and P.C.Ram, Unsteady magnetohydrodynamic fluid flow through a channel, J. Scientific Research XXVIII No, 2 (1978).

[13]. J.S, Waterhouse, and J.G.Kingston, .Plane magnetohydrodynamic flows with constantly inclined magnetic and velocity fields, J. Appl.Math, Phys. Vol.24 (1973), 653-658. 